\title{
Reliability and validity of a questionnaire for diagnosis of clinical gastric related vertigo: A Traditional Persian Medicine perspective and prevalence assessment
}

\author{
Maryam Monfared ${ }^{1,2}$, Alireza Karimi-Yazdi ${ }^{3}$, Fataneh Hashem-Dabaghian ${ }^{1,4}$, Mohammad Azizkhani ${ }^{5}$, Farshad \\ Amini-Behbahani ${ }^{1,6}$
}

\footnotetext{
${ }^{1}$ Research Institute for Islamic and Complementary Medicine, Iran University of Medical Sciences, Tehran, Iran

${ }^{2}$ M.D. and Ph.D. Candidate of Persian Medicine, School of Persian Medicine, Iran University of Medical Sciences, Tehran, Iran

${ }^{3}$ M.D. ENT, Associate Professor, Otorhinolaryngology Research Center, Tehran University of Medical Sciences, Tehran, Iran

${ }^{4}$ M.D. Community Medicine, Assistant Professor, School of Persian Medicine, Iran University of Medical Sciences, Tehran, Iran

${ }^{5}$ M.D. and Ph.D. Candidate of Persian Medicine, School of Persian Medicine, Tehran University of Medical Sciences, Tehran, Iran

${ }^{6}$ M.D. and PhD. of Persian Medicine, Assistant Professor, School of Persian Medicine, Iran University of Medical Sciences, Tehran, Iran
}

\section{Type of article: Original}

\begin{abstract}
Background: Chronic vertigo is a frustrating and expensive disease affecting individuals and society. Scientists of Traditional Persian Medicine (TPM) have a long held belief that there is a common type of vertigo originated from the stomach known as gastric related vertigo (GRV) and there are present, simple and low-cost treatments for GRV based on its categorization.

Objective: To develop a valid tool for assessing GRV and determining the prevalence of this type of vertigo.

Methods: In this cross-sectional study, a questionnaire was designed based on GRV indices. To determine the intra-rater reliability, a test- retest method was used and kappa coefficient was measured by Statistical Package for the Social Sciences software (SPSS, version 17). Content validity ratio (CVR) and content validity index (CVI) for each question were calculated by using Lawshe table. The reliable version of the questionnaire was assessed in a sample of 135 patients with a chronic true vertigo which lasts more than three months and aged between 18 and 65 years. This study was conducted in the outpatient clinics of several university hospitals in Tehran, Iran, between May 2016 and November 2017.

Results: A valid 30-item questionnaire with CVR more than 0.62 , CVI more than 0.78 and kappa coefficient more than 0.7 was the main achievement of this study, which can be useful for assessment of GRV in future clinical trials. The study showed that 98 participants $(72.59 \%)$ had at least one criterion of GRV.

Conclusion: According to the high prevalence of GRV among patients who suffer from chronic vertigo, more studies in this field including clinical trial are suggested.

Keywords: Vertigo; Traditional Medicine; Gastric Vertigo; Questionnaire
\end{abstract}

\section{Introduction}

Vertigo is a medical condition which is defined as a false sensation of spinning or moving in oneself or surroundings, and usually occurs with difficulties in walking, sweating, nausea and vomiting $(1,2)$. It is a common complaint in neurology, otolaryngology and general clinics. Some studies show a $7.4 \%$ lifetime prevalence of vertigo in adult

\section{Corresponding author:}

Associate Professor Dr. Farshad Amini-Behbahani. Tel: +98.9126088191, Email: aminibehbehani.f@iums.ac.ir Received: August 09, 2018, Accepted: December 17, 2018, Published: March 2019

iThenticate screening: December 22, 2018, English editing: December 30, 2018, Quality control: January 06, 2019

This article has been reviewed / commented by six experts

Funding / research project approval: Iran University of Medical Sciences (grant no. 9221309207)

Ethics approval: Iran University of Medical Sciences (Ref: IR.IUMS.REC.1395.9221309207, October, 08, 2016)

(C) 2019 The Authors. This is an open access article under the terms of the Creative Commons Attribution-NonCommercialNoDerivs License, which permits use and distribution in any medium, provided the original work is properly cited, the use is noncommercial and no modifications or adaptations are made. 
patients between 18-79 years and its prevalence and incidence during the year are $4.9 \%$ and $1.4 \%$ respectively (3). It is suggested that prevalence of vertigo may be similar to that of the USA in developing countries like Iran (4). In the elderly, fall and fear of falling will negatively influence the quality of life (5). Patients with episodic vertigo reported significantly better quality of life than patients with persistent vertigo (6). Chronic dizziness and vertigo can result in loss of function, fall, and injuries that can lead to nursing home placement, stroke, and death (7). There are several etiological factors for vertigo including benign paroxysmal positional vertigo (BPPV), Meniere's disease, labyrinthitis, vascular disorders in the elderly, craniovertebral junction disorders, migraines, brain tumors such as vestibular schwannoma, tumors in the cerebellum and brainstem, multiple sclerosis, head trauma and brain injury, focal seizure disorders, and toxin and drug exposures such as aminoglycoside antibiotics, carbon monoxide, anticoagulants, alcohol, or aspirin (1, 2, 8-11). Similarly, excessive motion, spinning with closed eyes, motion and sea sickness can cause physiologic vertigo $(2,12,13)$. Vertigo is usually classified into peripheral and central vertigo based on the location of the dysfunction in the vestibular pathway (14).

Most long-term pharmacological treatments used for prevention or treatment of vertigo have been associated with side effects, which makes some patients leave the treatment. Consequently, the individual's professional and social life is seriously affected by vertigo, and therefore, there is a tendency to use complementary and alternative therapies (15). Moreover, in conventional medicine, the concept of relationship between peripheral vertigo and gastrointestinal reflux disease has been mentioned and some potential mechanisms are proposed (16), this type of vertigo, is well discussed in Traditional Persian Medicine (TPM) textbooks and named as gastric related vertigo (GRV) which has simple and low-cost treatments with minimal side effects (17-24). Vertigo is widely studied by TPM scholars and is known as "Dowar". Scholars have described etiological factors, clinical manifestations and treatment strategies of vertigo in detail (17-24). In the TPM literatures, vertigo has been classified into two main groups:

1. Relating to external factors such as looking at a spinning object, turning around in a fast motion, exposure to a sudden coldness or heat that changes the temperament, head trauma, poisons and drug usage.

2. Relating to internal causes, which are divided into two subtypes:

a) Vertigo is caused by intracranial disorders according to four humors (black bile, yellow bile, phlegm, and blood) and the cerebral wind (Reeh), based on TPM, Reeh is a current flow with potential of affecting the normal brain functions.

b) Vertigo that is associated with different body organs such as stomach, uterus, kidney and bladder.

Any functional impairment in the above mentioned organs influence the brain and can cause vertigo. The stomach is the most important of these organs $(19,20)$. According to TPM literature, GRV is defined as a vertigo that is originated from gastric dysfunction or diseases; it can be caused by aggregation of the four humors and wind (as a blowing current flow which could cause fasciculation, spasms and flatulence) in the stomach. The clinical manifestations and treatment methods of this disease have been discussed in TPM textbooks in detail (18-25). However, to our knowledge, this type of vertigo has not been properly considered until now, so, this study is designed to introduce TPM concept of GRV and develop a standard tool for assessing and determining this type of vertigo.

\section{Material and Methods}

This cross-sectional study was conducted in the outpatient clinics of several university hospitals in Tehran, Iran, between May 2016 and November 2017 in two phases. In the first phase, we designed a preliminary self-administered questionnaire according to clinical manifestations of GRV and evaluated its validity and reliability. In the second phase the GRV was investigated among a sample of adult patients with diagnosis of true vertigo. The ethics committee of Iran University of Medical Sciences (Ref: IR.IUMS.REC.1395.9221309207, Date: October, 08, 2016) approved the ethics of the study.

\subsection{Phase 1: Developing the study tool and its validation \\ 2.1.1. Item Generation}

To assess GRV among the participants, we developed a questionnaire by reviewing the main and credible TPM textbooks (18-26). The textbooks of different periods were reviewed carefully by two experts in TPM with a focus on GRV, until data saturation was achieved. Those items which were persistent in the textbooks for identifying the symptoms of GRV were selected, according to each of the items resulting from the first stage, one statement which was appropriate for a self-report questionnaire was selected, and finally, we developed a preliminary self-administered questionnaire as closed-ended questions which could be answered with "Yes" or "No".

\subsubsection{Assessment of the face validity of statements}

In order to determine face validity, 26 patients with true vertigo who visited the outpatient clinic of otolaryngology in Amir Aalam Hospital of Tehran University of Medical Sciences were selected, they completed the preliminary 
questionnaire and wrote their opinions in terms of any obscurity, difficulty and appropriateness of the questions or answers.

\subsubsection{Assessment of the content validity of statements}

In this regard, our first expert panel including 10 TPM practitioners was asked to make their comments in terms of importance, significance and item allocation on the statements. In order to assess the content validity, the initial questionnaire was sent to the second expert panel that included 20 TPM practitioners who had at least 5 years of clinical experience, and were asked to write their opinions on the statements and suggest additional comments about the items if needed. Finally, 11 experts responded and returned the completed forms. Each form was sent to the experts via email along with some explanation about the content validity indices such as relevancy, clarity and comprehensiveness of the questionnaire. The experts were asked to determine whether each item is "essential" or "not essential, but useful" or "not relevant"; and to give a score on a 4-point scale that rated the clarity, relevance and appropriateness, for example, $1=$ not relevant, $2=$ somewhat relevant, 3 = quite relevant, $4=$ highly relevant. The validity of questionnaire was assessed by Lawshe table, also, content validity ratio (CVR) was calculated for each item based on this formula:

$\mathrm{CVR}=\frac{n_{e-N_{2}}}{N_{2}}$

$n_{e}=$ number of panel members that considered that question as essential

$N_{2}=$ total number of panel members divided by two (27).

Content validity index (CVI) was measured based on the Waltz and Basel method. According to this method, calculating the CVI for each item is done by computing the number of experts giving a rating of either 3 or 4 , divided by the number of experts (28). The statements with a CVR more than or equal to 0.62 indicated an acceptable agreement, a CVI more than or equal to 0.78 were retained as well.

\subsubsection{Assessment of the reliability}

To determine the intra-rater reliability, test- retest was conducted and kappa coefficient was measured by Statistical Package for the Social Sciences software (SPSS, version 17). A two-week interval was considered for the test- retest method. For evaluating this criterion, twenty patients with true vertigo in the outpatient clinic of Valiasr Hospital of Tehran University of Medical Sciences were asked to complete the questionnaire. The "Cohen's kappa coefficient" more than 0.7 were considered acceptable $(29,30)$. Test- retest method was chosen because some questions have two, and some have more than two options for answering. Also, only one independent concept was taken from each question, so, calculation of Cronbach's alpha was not necessary.

\subsection{Phase 2: Assessment of the prevalence of GRV}

\subsubsection{Study Design}

This cross-sectional study was conducted for evaluation of prevalence of GRV among a population of patients with vertigo; the study was performed in the otolaryngology outpatient clinics of Amir Aalam Hospital and Valiasr Hospital of Tehran University of Medical Sciences and Behesht Traditional Iranian Medicine outpatient clinic of Iran University of Medical Sciences. The data were collected between November 2016 and November 2017.

\subsubsection{Participants}

Among 600 patients in outpatient clinics of those university hospitals, with the chief complaint of dizziness during one year, 135 patients who have true vertigo were selected, the inclusion criteria were history of true vertigo with a feeling of spinning which lasts more than three months and aged between 18 to 65 years. Exclusion criteria were suffering from dizziness (not true vertigo with a feeling of spinning), having a history of otological surgery or head trauma.

\subsubsection{Sample size and statistical methods}

The sample size was calculated as 131 individuals based on the formula $n=z^{2} p(1-p) / d^{2}$; assuming type-1 error of 0.05 and frequency of GRV as 70\% that was calculated in a pilot study on 26 patients (31). Data were analyzed by SPSS version 17 (SPSS Inc., Chicago, Illinois, USA). The answer to each item of the questionnaire was considered as frequency and percentage.

\section{Results}

\subsection{Item generation}

Credible TPM textbooks were evaluated by the research team, considering Avicenna's Canon of Medicine as an original reference and all symptoms and signs related to GRV were extracted and classified. Two main groups of symptoms were gathered: those which show relativity of vertigo to the stomach and those which were useful for determining gastric problem. Then, these signs and symptoms were summarized as seven criteria, the results are shown in Table 1. 
Table 1. The number of individuals with the GRV signs

\begin{tabular}{|l|l|l|l|}
\hline \multicolumn{2}{|l|}{ The symptom group* } & $\mathrm{n}$ & $\%$ \\
\hline 1 & $\begin{array}{l}\text { Occurrence of vertigo or increase in severity of vertigo with satiation or overeating and the } \\
\text { presence of digestion weakness signs such as feeling of gastric heaviness or gastric reflux or burp, } \\
\text { having acidic taste or preference of spicy food, or drooling of saliva or suffering from flatus }\end{array}$ & 19 & 14.07 \\
\hline 2 & $\begin{array}{l}\text { Occurrence of vertigo or increase in severity of vertigo by hunger and relief by eating food with } \\
\text { the presence of hot humors' signs such as the bitterness of the mouth or feeling of dryness in the } \\
\text { mouth or excessive thirst and satiation of it with cold water, or bad odor from burping }\end{array}$ & 50 & 37.07 \\
\hline 3 & Occurrence or worsening of vertigo by stomach, abdominal and periumbilical pain & 21 & 15.5 \\
\hline 4 & $\begin{array}{l}\text { Occurrence or worsening of vertigo by eating some types of food with the presence of other } \\
\text { digestive symptoms such as flatus, reflux and burp }\end{array}$ & 57 & 42.2 \\
\hline 5 & Vertigo occurrence follows the occurrence of flatus & 17 & 12.6 \\
\hline 6 & Vertigo occurrence follows the occurrence of nausea & 21 & 15.5 \\
\hline 7 & Vertigo occurrence with or following the occurrence of vomiting & 36 & 26.6 \\
\hline 8 & Vertigo relief by vomiting & 22 & 16.29 \\
\hline
\end{tabular}

*Data are not exclusive

\subsection{Content and face validity results}

The extracted indices for designing the preliminary questionnaire with 35 questions and the results of CVI and CVR calculation are shown in Table 2, each recent question had CVI between 0.70 and 1.00 and therefore, most of the TPM experts had agreement with items and their related questions. The result of content validity analysis showed that 4 items did not have acceptable agreement.

Table 2. The result of content validity assessment of the items of data collection form

\begin{tabular}{|c|c|c|c|c|}
\hline No. & Item & $\mathrm{CVI}$ & CVR & Accepted \\
\hline 1 & Do you suffer from temporal tinnitus? & 0.8 & 0.78 & Yes \\
\hline 2 & Whether vertigo occurs when you are hungry & 1.0 & 1.0 & Yes \\
\hline 3 & Whether vertigo severity increases when you are hungry & 1.0 & 0.64 & Yes \\
\hline 4 & Whether eating food and satiety affect the vertigo severity? & 1.0 & 1.0 & Yes \\
\hline 5 & $\begin{array}{l}\text { Whether you can digest junk foods such as Pizza or "sheep foot pate" (a high-fat } \\
\text { high-cholesterol Iranian traditional food) easily }\end{array}$ & 1.0 & 0.82 & Yes \\
\hline 6 & Whether you feel heavy stomach after eating food (without overeating) & 1.0 & 1.0 & Yes \\
\hline 7 & Whether your saliva increases when you are hungry & 1.0 & 0.64 & Yes \\
\hline 8 & Whether you suffer from drooling while sleeping & 0.9 & 1.0 & Yes \\
\hline 9 & Whether you suffer from flatus & 1.0 & 1.0 & Yes \\
\hline 10 & Whether you suffer from stomach rumbling or growling & 0.9 & 0.45 & No \\
\hline 11 & Whether you first suffer from flatus and then vertigo occurs & 1.0 & 0.78 & Yes \\
\hline 12 & Whether you suffer from gastric reflux & 0.9 & 1.0 & Yes \\
\hline 13 & Whether you burp frequently & 0.9 & 1.0 & Yes \\
\hline 14 & Whether your burp has a bad smell or acidic taste & 0.9 & 1.0 & Yes \\
\hline 15 & Which taste do you prefer more (spicy, sour, salty, sweet or bitter)? & 0.8 & 0.82 & Yes \\
\hline 16 & Whether when vertigo occurs, you feel a stomach ache & 0.9 & 1.0 & Yes \\
\hline 17 & Whether at the time of vertigo, you feel an abdominal pain or a pain around the navel & 0.9 & 1.0 & Yes \\
\hline 18 & Do you have a small appetite & 0.9 & 0.56 & No \\
\hline 19 & Do you feel the change in your appetite? & 0.8 & 0.27 & No \\
\hline 20 & Whether you have nausea & 1.0 & 0.82 & Yes \\
\hline 21 & Whether you have nausea and then the vertigo occurs & 0.9 & 1.0 & Yes \\
\hline 22 & Whether you vomit while having vertigo & 1.0 & 0.9 & Yes \\
\hline 23 & Whether your vertigo diminishes if you vomit & 0.9 & 1.0 & Yes \\
\hline 24 & $\begin{array}{l}\text { What is the color of your content of vomiting (yellow, white, foamy, dark or eaten } \\
\text { food)? }\end{array}$ & 0.8 & 0.78 & Yes \\
\hline 25 & Whether you suffer from bitterness of the mouth & 0.9 & 1.0 & Yes \\
\hline 26 & Whether you suffer from excessive thirst & 0.9 & 1.0 & Yes \\
\hline 27 & Does cold or warm water satisfy your thirst? & 1.0 & 0.78 & Yes \\
\hline
\end{tabular}


http://www.ephysician.ir

\begin{tabular}{|l|l|l|l|l|}
\hline 28 & Whether you suffer from dryness of mouth & 1.0 & 0.82 & Yes \\
\hline 29 & Whether you suffer from frequent hiccups & 0.9 & 0.78 & Yes \\
\hline 30 & Whether you suffer from the feeling of stretch or pain below the ribs & 0.8 & 0.45 & No \\
\hline 31 & Whether you suffer from constipation & 1.0 & 1.0 & Yes \\
\hline 32 & Whether vertigo occurs after constipation (no passing of stool for two or three days) & 1.0 & 1.0 & Yes \\
\hline 33 & Whether vertigo occurs after eating food (within two hours) & 0.7 & 0.78 & Yes \\
\hline 34 & Whether vertigo occurs when you wake up from sleep & 1.0 & 1.0 & Yes \\
\hline 35 & $\begin{array}{l}\text { Is there any food item that when you eat it, it triggers vertigo (such as garlic, raw } \\
\text { onion, pepper or fatty food)? }\end{array}$ & 1.0 & 1.0 & Yes \\
\hline
\end{tabular}

\subsection{Items reliability analysis}

Twenty participants were invited to accomplish this assessment; in conclusion, the result of reliability test showed that items related to drooling at night, change in appetite, constipation and contents of vomiting did not have Cohen's kappa coefficient more than 0.7 and finally were deleted.

\subsection{Assessing the prevalence of GRV}

During the study period, from a total of 600 patients referring with vertigo, 135 patients with chronic true vertigo were eligible and willing to participate in the study. Table 3 shows the characteristics of the participants, mean age of them was 45.2 years ( \pm 11.2 days), most of the participants were females $(73.3 \%)$ and married $(85.9 \%)$, history of disorder among patients included high blood pressure (7.6\%), thyroid disorder (6.4\%), dyslipidemia (5.8\%) and 40.9\% did not have any history of a disease. Five patients had BPPV, 20 patients had Meniere's disease or endolymphatic hydrops and others had no definite diagnosis. All participants had a minimum of three months history of recurrent vertigo, 129 patients (95\%) had gastrointestinal symptoms or signs, and 98 patients $(72.59 \%)$ had at least one criterion of GRV.

Table 3. Characteristics of participant

\begin{tabular}{|c|c|c|c|}
\hline \multicolumn{2}{|c|}{ Characteristics } & $\mathrm{n}$ & $\%$ \\
\hline \multicolumn{4}{|c|}{ Age Mean: 45.2, SD: 11.2 (year) } \\
\hline \multirow{2}{*}{ Gender } & Male & 36 & 26.7 \\
\hline & Female & 99 & 73.3 \\
\hline \multirow[t]{5}{*}{ Marital status } & Single & 14 & 10.4 \\
\hline & Married & 116 & 85.9 \\
\hline & Divorced & 2 & 1.5 \\
\hline & Widow & 1 & 0.7 \\
\hline & Non-response & 2 & 1.5 \\
\hline \multirow{15}{*}{ History of disease* } & No disease & 70 & 40.9 \\
\hline & High blood pressure & 13 & 7.6 \\
\hline & Thyroid disorder & 11 & 6.4 \\
\hline & Dyslipidemia & 10 & 5.8 \\
\hline & Anemia & 9 & 5.3 \\
\hline & Diabetes Mellitus & 9 & 5.3 \\
\hline & Cardiovascular problems & 9 & 5.3 \\
\hline & Nervous and mental problems & 6 & 3.5 \\
\hline & Musculoskeletal problems & 6 & 3.5 \\
\hline & Fatty liver disease & 5 & 2.9 \\
\hline & Renal problems & 3 & 1.8 \\
\hline & Gastrointestinal problems & 3 & 1.8 \\
\hline & Pulmonary problems & 2 & 1.2 \\
\hline & Migraine & 2 & 1.2 \\
\hline & Other & 13 & 7.6 \\
\hline
\end{tabular}

*data are not exclusive

In patients with BPPV, four patients did not have any criteria of GRV and one patient had two criteria, suffering from two types of vertigo including BPPV and vertigo, which became worse when the patient was hungry and after eating rich fatty foods. The individual also had flatus and sensation of bitterness and dryness in her mouth. In patients with Meniere's disease or endolymphatic hydrops (twenty patients), 15 of them had GRV criteria. Among all participants, 
only six patients (4.4\%) had no gastric signs mentioned in the questionnaire. In this study, the occurrence or worsening of vertigo by eating has the highest frequency. The details are as follows: vertigo after eating some types of food and the presence of flatulence, reflux and burp were found in 57 cases $(42.2 \%)$, vertigo associated with hunger and relief after eating in 50 participants (37.07\%) and vertigo with satiation or overeating in 19 participants $(14.07 \%)$ were observed, 21 participants (15.5\%) had vertigo in association with abdominal pain, 36 participants $(26.6 \%)$ had vertigo along with vomiting and in 22 cases, (16.29\%) vertigo diminished after vomiting. Also, occurring or worsening of vertigo associated with constipation has been observed in 36 patients, although the question about constipation was not deemed reliable. The frequency of GRV criteria among the study population is presented in Table 1 . In 98 patients with GRV, 19 patients (19.38\%) had just one criterion, 41patients (41.83\%) had two criteria, 19 patients (19.38\%) had three criteria, 12 patients $(12.24 \%)$ had four criteria, five patients $(5.1 \%)$ had five criteria and two patients $(2.04 \%)$ had six GRV criteria.

\section{Discussion}

TPM has a holistic approach in the diagnosis and treatment of diseases, and considers interrelated effects of different body organ dysfunction in other organs (18-26). There is a bidirectional relationship between the brain and stomach, wind and vapors of humors can rise upward and penetrate the brain and cause certain diseases, vertigo is one of them $(19,20)$. It is believed that, vapors come to the brain through the membranes and nerves, and then influence its function. In this process, it is suggested that some debility in the brain can cause more damage by these vapors. Modern medicine is not familiar with "wind" and "vapors" as effective factors in the body. As a hypothesis, we suggest increased gastric bioelectromagnetic pulses or a deficit in electrical resistance pathway of these pulses toward the brain as an electrical interfering mechanism with vestibular pulses. Also, releasing of unknown chemicals by the stomach could be another explanation for GRV. There is evidence of a relationship between the brain and stomach; types of gastric-related headaches have also been reported in addition to GRV (32). In recent studies, a relationship between the brain and the digestive tract has been considered though gut-brain axis, some diseases such as Alzheimer's disease (33), anxiety and depression (34), multiple sclerosis, autism, stress (35) and dementia (36) which are recently proposed "may begin in the gut". A noble questionnaire for assessing GRV with an acceptable reliability and validity was the first achievement of this study. This self-answering questionnaire as a checklist provides sufficient information for diagnosis of GRV in TPM clinics. Despite having less accuracy, it can help clinicians to detect more particular original diseases in the stomach from the viewpoint of TPM, and so, management of GRV. Interpretation of the questionnaire depends on understanding the content of the vertigo chapters in TPM textbooks especially Avicenna's Canon of Medicine. The final decision is up to the clinician.

The second result of research was high prevalence of GRV among patients whom suffer from chronic vertigo. If TPM assurances in simple treatment of GRV are legitimate, a significant improvement in chronic vertigo is expected. Considering 37 and 41 percent prevalence of symptom groups $2 \& 4$ (Table 1), future suggested treatment should focus on presence of choleric and phlegm in the stomach. In other words, prescribing concentrated pomegranate juice will help at least $37 \%$ of patients and avoiding drinking fluids with food, chewing foods well and prescription of quince jam after each meal will help $41 \%$ of patients who suffer from chronic vertigo. More accurate and successful treatment will depend on the clinician's skill to choose proper single or combined treatment.

There are some limitations to our study: 1) the main limitation of this study was the lack of clinical trials in this field, meaning we were not able to determine the weighing of each defined symptom group exactly. In other words, we are not able to say which symptoms group is more important to determine GRV. It is possible the nomination of criteria for these symptoms groups needs more deliberation for future developed studies, 2) another limitation was the lack of attention to the symptoms by patients, some of patients did not remember some symptoms in their first visit and for solving this problem we rechecked the symptoms by the second visit or telephone calls, 3 ) in this study, the concept of temperament has not been considered; temperament may play a key role in GRV, 4) clinical manifestations of GRV have not been also compared with current medical resources, 5) there is no gold standard for diagnosis of GRV, and experts in this field of Traditional Persian Medicine were rare and inaccessible.

\section{Conclusions}

The main achievement of this study was a 30-item valid questionnaire as a checklist for detecting GRV which demonstrated high prevalence of GRV among patients who suffer from chronic vertigo. This is a new aspect that can have great clinical implications in treatment of chronic vertigo, with simple and low cost TPM suggested treatments for GRV. It is suggested clinical trials be rendered in this field. Also, further evaluation of GRV among other kinds of vertigo as a supplementary study is suggested to be accomplished. 


\section{Acknowledgments:}

The present study is part of a PhD thesis in Traditional Persian Medicine by Dr. M. Monfared, which was supported by funding (grant no. 9221309207) from the deputy of research and technology of Iran University of Medical Sciences, Tehran, Iran. Authors wish to thank Roxana Mirkazemi and Bahare Dadmehr for assistance in editing. We would also like to thank the members of the expert's panel: Dr. Effat Jafari-Dehkordi, Dr. Somaye Mahroozade, Dr. Elham Akhtari and Dr. Hoorieh Mohammadi Kenari from Tehran University of Medical Sciences, Dr. Sayed Mohammad Ali Soroushzadeh, Mr. Mohammad Ebadiani and Mr. Esmaeil Nazem from Tehran University of Medical Sciences, Dr. Mohammad Ali Zareian, Dr. Mahboobeh Abrishamkar, Dr. Mamak Hashemi, Dr. Fatemeh Mohammadi, Dr. Jale Aliasl, Dr. Hale Tajadini, Dr. Aisan Nozad, Dr. Nafiseh Hosseini Yekta, Dr. Nasrin Baery, Dr. Fariba Ghorbani, Dr. Fatemeh Mohamadi Sorme, Dr. Maryam Shayesteh and Dr. Vahid Aliannezhadi, all of whom helped us in this research.

\section{Conflict of Interest:}

There is no conflict of interest to be declared.

\section{Authors' contributions:}

All authors contributed to this project and article equally. All authors read and approved the final manuscript.

\section{References:}

1) Flint PW, Haughey BH, Lund VJ, Thomas JR, Niparko JK, Robbins KT, et al. Cummings OtolaryngologyHead and Neck Surgery E-Book. Saunders, an imprint of Elsevier Inc. 2015.

2) Hogue JD. Office evaluation of dizziness. Primary Care: Clinics in Office Practice, 2015; 42(2): 249-58. doi: 10.1016/j.pop.2015.01.004.

3) Von Brevern M, Neuhauser H. Epidemiological evidence for a link between vertigo and migraine. Journal of Vestibular Research. 2011; 21(6): 299-304. doi: 10.3233/VES-2011-0423.

4) Tunes C, Goplen FK, Wester K. Clinical Presentation, Symptoms, and Complaints: Dizziness/Vertigo. Arachnoid Cysts, Clinical and Surgical Management. Elsevier. 2018; 2-9. doi: 10.1016/B978-0-12-8143780.00002-9.

5) Silva AL, Ferreira LMBM, Freitas RVM, Lima KC, Guerra RO, Ribeiro KMOBF. Quality of life in the institutionalized elderly with dizziness complaint: a cross-sectional study. Rev CEFAC. 2018; 20(2): 22837. doi: 10.1590/1982-021620182023017.

6) Grill E, Akdal G, Becker-Bense S, Hübinger S, Hübinger S, Huppert D, et al. Multicenter data banking in management of dizzy patients: first results from the Dizzy Net registry project. Journal of Neurology. 2018; 265(1): 3-8. doi: 10.1007/s00415-018-8864-1.

7) Hilas O. Management of Dizziness and Vertigo. US Pharm. 2012; 37(1): 30-3.

8) Post RE, Dickerson LM. Dizziness: a diagnostic approach. Am Fam Physician. 2010; 82(4): 361 -8. PMID: 20704166.

9) Bronstein A. Oxford textbook of vertigo and imbalance: OUP Oxford; 2013.

10) Goebel JA. Practical management of the dizzy patient: Lippincott Williams \& Wilkins; 2008.

11) Wicks R. Alternobaric vertigo: an aeromedical review. Aviation, space, and environmental medicine, 1989; 60(1): 67-72. PMID: 2647073.

12) Hankey G, Wardlaw J. Clinical neurology. London: Manson; 2008.

13) Harrison C, Falvo D, Weiss V, Holland BE. Medical and psychosocial aspects of chronic illness and disability: Jones \& Bartlett Learning; 2017.

14) Wippold F, Turski P. Vertigo and hearing loss. AJNR Am J Neuroradiol. 2009; 30(8): 1623-5. PMID: 19749077.

15) Ferley JP, N Poutignat, Y Azzopardi, M Charrel. Homeopathy trials used and not used in Egger/Shang's meta-analysis. J Altern Complement Med. 2005; 11(1): 155-60.

16) Viliušytė E, Macaitytė R, Vaitkus A, Rastenytė D. Associations between peripheral vertigo and gastroesophageal reflux disease. Medical Hypotheses. 2015; 85(3): 333-5. doi: 10.1016/j.mehy.2015.06.007.

17) Heravi M. Bahroljavaher. Qom: Ehyaye Tebbe Tabie; 2008.

18) Majūsī-Ahvazi A. Kāmil al-Sināa al Tibbiya(The Perfect Book of the Art of Medicine). Qom: Ehya-e Tebbe Tabiee Institution; 2008.

19) Ibn-e-sina(Avicenna) A. Al-Qānūn fĩ al-Tibb (Canon of Medicine). Beirut: Dare Ehyae al-Torathe al-Arabi; 2005. 
20) Jorjani E. Zakhireye Kharazmshahi (Treasure of the Khwarazm Shah). Qom: Ehya-e Tebb-e Tabiee Institution; 2012.

21) Kermani N. Sharh al-Asbab wa al-Alamat (Explanation of causes and signs). Qom: Ehya-e Tebb-e Tabiee Institution; 2012.

22) Aghili S. (Moalejat-e-Aghili) Aghili's Treatments. Tehran: Research Institute for Islamic and Complimentary Medicine; 2008.

23) Arzani M. Tebb-e-Akbari (Akbari's Medicine). Qom: Ehya-e Tebb-e Tabiee Institution; 2008.

24) Chashti M. Exir-e Azam (Azam's Elixir). Tehran: Iran University of Medical Science, Institute for Islamic and Complementary Medicine; 2007.

25) Ibn-e-Nafiss. Almoojaz fit-teb. Beirut: Dar Al-kotob Al-ilmiyah Publication; 2004.

26) Akhawayni-Bukhari A. Hidayat al-Mutaallimin fi al-Tibb (A Guide to Medical Students). Mashhad: Mashhad University Press; 1992.

27) Lawshe CH. A quantitative approach to content validity, Personal Psychology, 1975; 28(4): 563-575. doi: 10.1111/j.1744-6570.1975.tb01393.x.

28) Waltz CF, Bausell BR. Nursing research: design statistics and computer analysis. 1981.

29) Downing SM. Reliability: on the reproducibility of assessment data. Medical Education. 2004; 38(9): 100612. doi: 10.1111/j.1365-2929.2004.01932.x.

30) Gwet KL. Handbook of inter-rater reliability: The definitive guide to measuring the extent of agreement among raters. Advanced Analytics LLC. 2014

31) Daniel WW. A foundation for analysis in the health sciences. Biostatistics. Toronto, John Wiley \& Sons; 1999.

32) Noghani MT, Rezaeizadeh H, Fazljoo SM, Keshavarz M, Gastrointestinal Headache; a Narrative Review. Emergency. 2016; 4(4): 171. PMID: 27800536, PMCID: PMC5007907.

33) $\mathrm{Hu} \mathrm{X}$, WangT, Jin F. Alzheimer's disease and gut microbiota. Science China Life Sciences. 2016; 59(10): 1006-23. doi: 10.1007/s11427-016-5083-9.

34) Bravo JA, Julio-Pieper M, Forsythe P, Kunze W, Dinan TG, Cryan JF, et al. Communication between gastrointestinal bacteria and the nervous system. Curr Opin Pharmacol. 2012; 12(6): 667-72. doi: 10.1016/j.coph.2012.09.010.

35) Ader R, Cohen N, Felten DL. Brain, behavior, and immunity. Brain Behav Immun. 1987; 1(1): 1-6. doi: 10.1016/0889-1591(87)90001-8.

36) Maqsood R, Stone TW. The gut-brain axis, BDNF, NMDA and CNS disorders. Neurochem Res. 2016; 41(11): 2819-35. doi: 10.1007/s11064-016-2039-1. 